\title{
PREVALÊNCIA DAS LESÕES MÚSCULO-ESQUELÉtiCAS RELACIONADAS COM O TRABALHO DOS ENFERMEIROS: REVISÃO INTEGRATIVA
}

Prevalence of work-related musculoskeletal disorders in nurses: integrative review

Prevalencia de trastornos musculoesqueléticos relacionados con el trabajo en los enfermeros: revisión integrativa

Liliane Castelôa*, Sónia Luís ${ }^{* *}$, Tiago Romeiro***, Isabel Oliveira ${ }^{* * *}$

\section{RESUMO}

Enquadramento: a prática de enfermagem está relacionada com atividades que requerem solicitação músculo-esquelética constante e desajustada, como consequência do desequilíbrio entre o profissional/equipamento/doente, levando os profissionais a adotarem posturas incorretas e prejudiciais à sua própria saúde. Objetivo: conhecer a prevalência das lesões músculo-esqueléticas relacionadas com o trabalho (LMERT) nos enfermeiros. Metodologia: para responder ao objetivo deste estudo realizou-se uma revisão integrativa, com uma pesquisa nas bases de dados Medline, SciELO e CINHAL, com os descritores "nurses", "musculoskeletal diseases" e "prevalence", combinados com o operador booleano "AND", dos estudos de prevalência publicados entre 2012 e 2017. Dos 174 estudos identificados, 24 foram incluídos para a revisão. Resultados: verifica-se elevada prevalência de LMERT, variando de 25 a $98 \%$, destacando-se a lesão da coluna lombar. Conclusão: as LMERT têm um impacte negativo na vida pessoal, profissional e social do enfermeiro. A investigação nesta área deverá ser dirigida para a interpretação dos fatores causais e implementação de medidas preventivas/corretivas e a capacitação das instituições para a importância de ações a nível preventivo, comportamental e instituição de medidas ergonómicas.

Palavras-chave: enfermeiros; lesões músculo-esqueléticas; prevalência

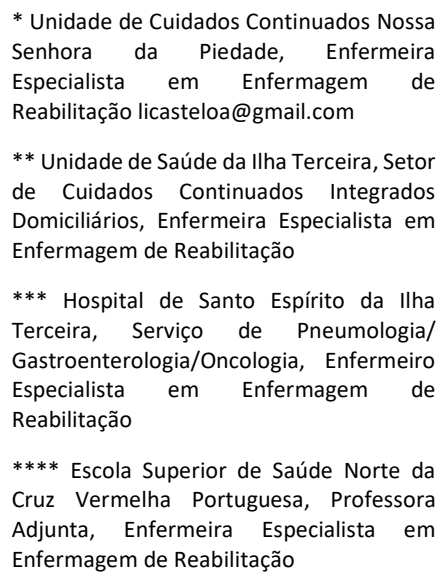

Como Referênciar:

Castelôa, L.; Luís, S.; Romeiro, T.; \& Oliveira, I. (2019). Prevalência das lesões músculoesqueléticas relacionadas com o trabalho dos enfermeiros: revisão integrativa. Revista de Investigação \& Inovação em Saúde, 2(1), 63-74

\section{ABSTRACT}

Framework: nursing practice is related to activities that require constant and misaligned musculoskeletal solicitation as a consequence of the imbalance between the professional / equipment/patient, leading professionals to adopt incorrect and harmful postures to their own health. Objective: to know the prevalence of work-related musculoskeletal injuries (WRMSD) in nurses. Methodology: an integrative review was conducted with a search in Medline, SciELO and CINHAL databases, with the descriptors "nurses", "musculoskeletal diseases" and "prevalence", combined with the Boolean operator "AND", from prevalence studies published between 2012 and 2017. Of the 174 studies identified, 24 were included for review. Results: there is a high prevalence of WRMSD, ranging from 25 to $98 \%$, especially lumbar spine injury. Conclusion: WRMSD has a negative impact on nurses' personal, professional and social life. Research in this area should be directed to the interpretation of causal factors and implementation of preventive / corrective measures and institutions' capacitation for the importance of preventive, behavioural and ergonomic measures.

Keywords: nurses; musculoskeletal disorders; prevalence

\section{RESUMEN}

Encuadramiento: la práctica de enfermería está relacionada con actividades que requieren una solicitud músculo-esquelética constante y desajustada, como consecuencia del desequilibrio entre el profesional / equipo / enfermo, llevando a los profesionales a adoptar posturas incorrectas y perjudiciales a su propia salud. Objetivo: conocer la prevalencia de los trastornos musculoesqueléticos relacionadas con el trabajo (TMERT) en los enfermeros. Metodología: una revisión integrativa de realizó con una búsqueda en las bases de datos Medline, SciELO y CINHAL, con los descriptores "nurses", "musculoskeletal diseases" y "prevalence", combinados con el operador booleano "AND, de los estudios de prevalencia publicados entre 2012 y 2017. De los 174 estudios identificados, 24 fueron incluidos para la revisión. Resultados: se destaca la elevada prevalencia de TMERT, variando de 25 a $98 \%$, destacándose la lesión de la columna lumbar. Conclusión: los TMERT tienen un impacto negativo en la vida personal, profesional y social del enfermero. La investigación en esta área deberá ser dirigida a la interpretación de los factores causales e implementación de medidas preventivas / correctivas y la capacitación de las instituciones para la importancia de acciones a nivel preventivo, comportamental e institución de medidas ergonómicas.

Palabras clave: enfermero; trastornos músculo-esqueléticos; prevalencia 


\section{INTRODUÇÃO}

Entendem-se por lesões músculo-esqueléticas relacionadas com o trabalho (LMERT) todas as lesões de músculos, tendões, nervos e ligamentos decorrentes de atividade laboral (Lelis, Battaus, Freitas, Rocha, Marziale \& Robazzi, 2012). Podem ocorrer de forma isolada ou em várias destas estruturas em simultâneo, bem como podem ser acompanhadas ou não de outros sintomas (Lelis et al., 2012). São frequentes nos membros superiores, região escapular e cervical, levando por vezes a incapacidades laborais temporárias ou permanentes (Lelis et al., 2012). Segundo Ranney (2000) aparecem em virtude do desequilíbrio na relação entre as exigências físicas contínuas do trabalho e a adaptação da região afetada, consequência da falta de descanso, dando origem a estados patológicos do sistema músculo-esquelético. O enfermeiro desenvolve a sua atividade num contexto laboral que o sujeita a um elevado risco para a sua saúde, com elevada solicitação física da musculatura da coluna vertebral, membros superiores e inferiores, como consequência de uma desadequação entre profissional/equipamento/doente adotando posturas prejudiciais e está ainda exposto a fatores de risco individuais, psicossociais, biomecânicos e organizacionais (Long, Bogossian, \& Johnston, 2013). No seio destes fatores insere-se ainda o stress, fruto da relação psicoemocional enfermeiro-doente, da limitação na capacidade de decisão sobre o trabalho, das condições inadaptadas para trabalhar, da solicitação física consequência da falta/distribuição ineficaz de recursos humanos, do excesso de carga horária e do trabalho por turnos (Lelis et al., 2012).

As LMERT são reconhecidas como resultado da exposição aos fatores de risco profissional há muitos anos, no entanto, só nas últimas décadas se manifestou o interesse neste âmbito, sobretudo na sua prevenção (Serralheira \& Uva, 2007), o que se compreende à luz dos diversos estudos nacionais e internacionais que dão enfase às repercussões negativas na produtividade, assiduidade e qualidade de vida que resultam das mesmas (Jerónimo \& Cruz, 2014). Considerando a infinidade de estudos sobre LMERT já publicados até ao momento (Davis \& Kotowsky, 2015; Lelis et al., 2012), uma compreensão geral da sua prevalência pode levar a uma adequada priorização das necessidades de investigação, que permitirão fundamentar a relevância de programas de prevenção das LMERT nos enfermeiros. Para tal, e com o intuito de atualizar a evidência disponível, propõe-se conhecer a prevalência das LMERT nos enfermeiros fornecendo evidência que permita dimensionar o problema. Assim, realizou-se uma revisão integrativa cujo objetivo é conhecer a prevalência das LMERT nos enfermeiros. A questão de investigação que norteou a pesquisa foi:" Qual a prevalência das lesões músculoesqueléticas relacionadas com o trabalho nos enfermeiros?" Procedimentos Metodológicos de Revisão. Para responder à questão de investigação, uma crítica e extensa análise de um conjunto de publicações foi realizado de acordo com os procedimentos metodológicos de uma revisão integrativa (Souza, Silva \& Carvalho, 2010). Uma vez identificada a área de interesse, foi formulada a questão de partida, para o qual se recorreu-se ao anagrama PIO que define, segundo Galvão \& Pereira (2014), a população (enfermeiros); a intervenção ou exposição (lesões musculoesqueléticas) e o desfecho, do inglês outcome (a prevalência). Durante o mês de dezembro de 2017 efetuou-se a pesquisa nas subsequentes bases de dados científicas: Medline, através da PubMed; Scientific Electronic Library Online 
(SCIELO) e Cumulative Index to Nursing and Allied Health Literature (CINAHL), definindo-se como critérios de inclusão os estudos de prevalência de LMERT nos enfermeiros, publicados em português, inglês, espanhol e francês, com texto integral acessível e data de publicação de 2012 a 2017, no sentido de conhecer a evidência mais recentemente produzida. Os critérios de exclusão definidos foram: estudos que não fossem de prevalência, que não apresentassem dados específicos para enfermeiros, que não estivessem relacionados com LMERT ou que fizessem a correlação com outras situações, revisões sistemáticas, atas, auditorias, teses, dissertações e outras provas académicas. Para a pesquisa foram usados os descritores do Medical Subject Headings, "nurses" e "prevalence" e o descritor "musculoskeletal diseases", que não sendo descritor $\mathrm{MeSH}$, surge como o termo mais comummente utilizado na denominação do fenómeno em estudo. Estes termos foram utilizados com o operador booleano "AND", compondo a seguinte fórmula: "musculoskeletal diseases" AND "nurses" AND "prevalence". Dois autores (LC e TR) realizaram a seleção dos artigos para a revisão sistemática da literatura. O processo de seleção encontra-se esquematizado na figura I, tendo sido realizada de acordo com o diagrama de fluxo PRISMA (Moher et al., 2015).

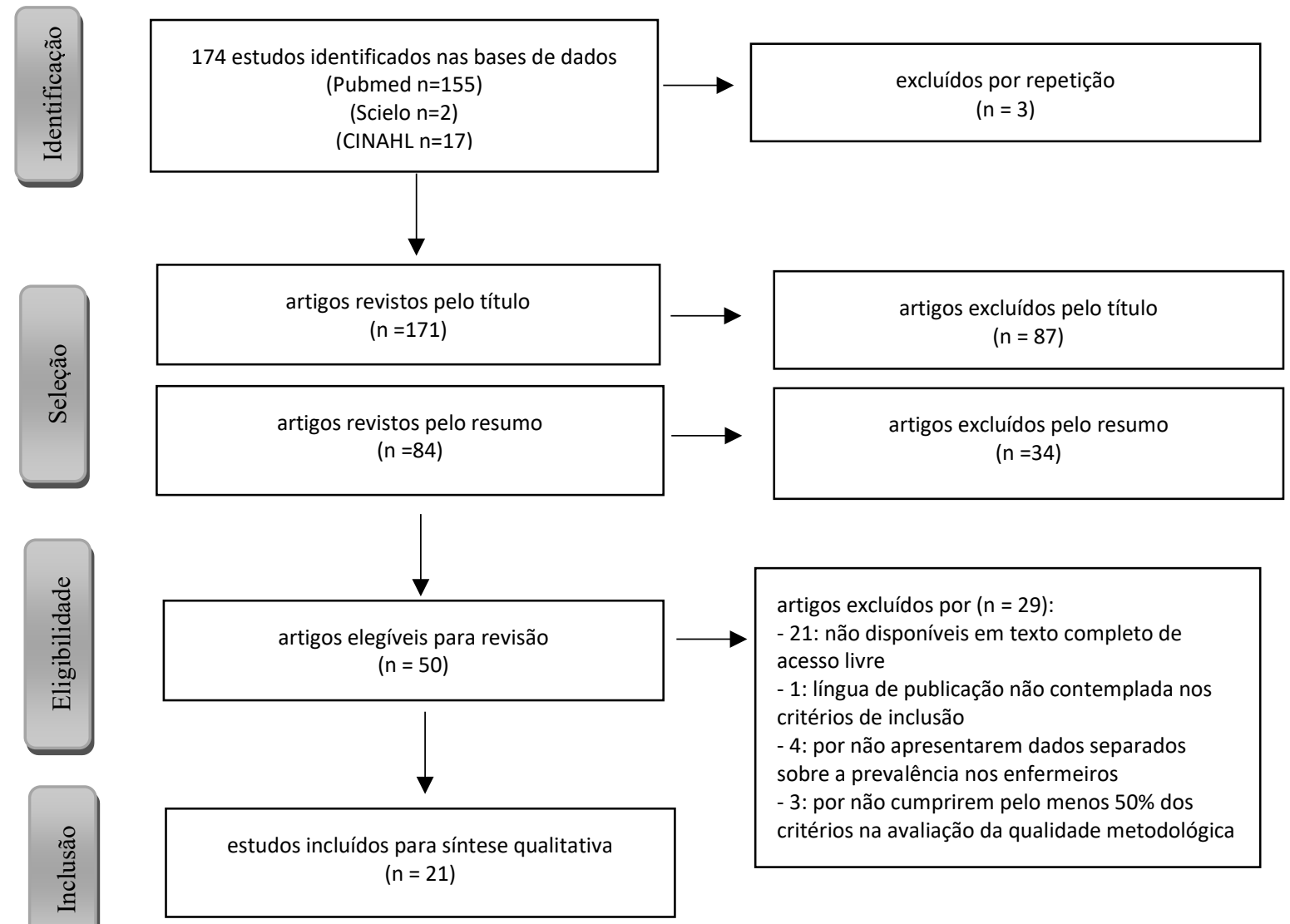

Figura 1

Seleção dos artigos para revisão sistemática da literatura 
Numa primeira pesquisa, com a aplicação dos limitadores "5 years", "Full Text" e "Humans" na Medline, identificaram-se 155 estudos, na SciELO identificaram-se 2 estudos e na CINHAL limitando a texto completo identificaram-se 17 estudos, perfazendo 174, sendo que 3 estudos da CINHAL foram eliminados por serem repetidos, identificandose 171 artigos. Na primeira fase de seleção excluíramse, pela leitura do título, 84 da Medline, 1 da Scielo e 2 da CINHAL, essencialmente por não estarem relacionados com a questão de investigação. Numa segunda seleção, feita pela leitura do resumo, foram excluídos 33 da Medline e 1 da CINHAL, por não apresentarem dados isolados para a população em estudo, não apresentarem dados de LMERT e por não serem estudos de prevalência, tendo ficado um total de 50 estudos. Destes, 26 foram excluídos por não cumprirem com alguns critérios de inclusão, nomeadamente 21 da Medline por não fornecerem texto integral acessível, 1 da CINHAL por não corresponder aos idiomas estipulados e 4 por não corresponderem a estudos de prevalência em enfermeiros (2 Medline e 2 da CINHAL), obtendo-se nesta fase 24 artigos para síntese qualitativa.

É de salientar que a maior parte dos estudos encontrados foram de prevalência, porém alguns eram em amostras multiprofissionais, não fornecendo resultados por grupo profissional.
Na fase final, todos os artigos (24) foram submetidos a avaliação da qualidade metodológica tendo por base os seguintes critérios: 1) representatividade da amostra, 2) recrutamento apropriado, 3) dimensão da amostra adequada, 4) descrição adequada de sujeitos e contextos, 5) abrangência da análise dos dados a toda a amostra, 6) objetividade dos critérios de medida da condição clínica em estudo, 7) confiabilidade da medida utilizada, 8) análise estatística apropriada, 9) identificação dos fatores confundentes e 10) critérios objetivos para a identificação de subpopulações (Briggs, 2017). A maior parte dos estudos estavam em conformidade com os critérios que definem a qualidade metodológica, sendo que apenas se excluíram 3 (1 da Medline e 2 da CINHAL) por não obedecerem a pelo menos $50 \%$ dos critérios. Assim, foram então encontrados um total de 21 artigos (14 da Medline, 1 da Scielo e 6 da CINHAL) que foram analisados integralmente e incluídos na revisão. A extração de dados obedeceu à matriz da tabela I e foi realizada por dois autores (TR e SS).

\section{RESULTADOS}

Na tabela 1 apresentam-se os artigos selecionados, para a revisão sistemática da literatura, destacando os seguintes aspetos: autor e ano, população-alvo/ amostra de enfermeiros, método de recolha de dados e resultado da prevalência. 
Tabela 1

\section{Caraterísticas dos estudos incluídos para revisão}

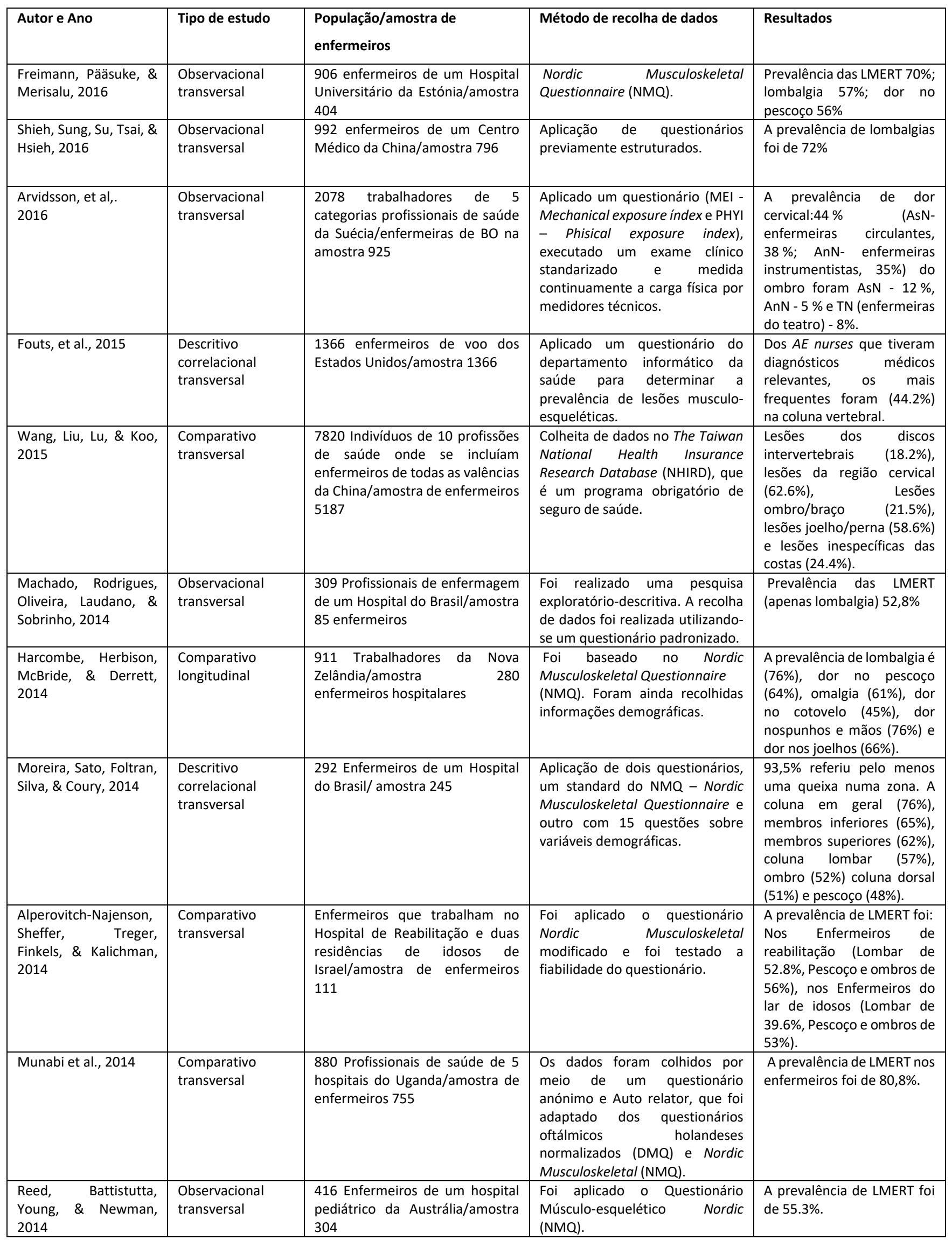




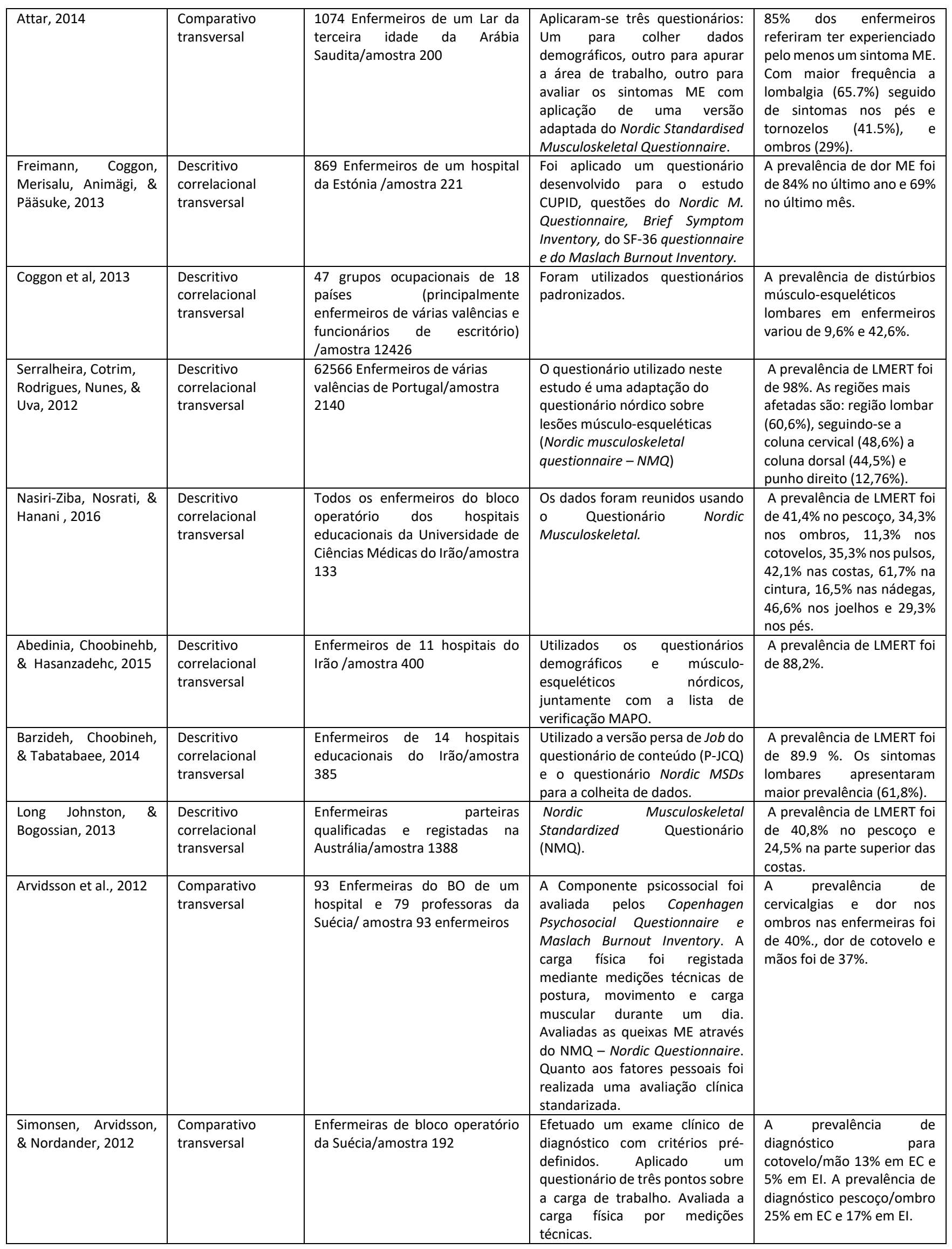


O número total de enfermeiros incluídos nestes estudos foi de 28036 , de diferentes países e inseridos em contextos laborais muito diversificados. Além disso, há estudos que permitem comparar as percentagens de LMERT dos enfermeiros com outros profissionais de saúde ou outras profissões que não se insiram no âmbito dos cuidados de saúde. Neste sentido, os enfermeiros revelaram maiores taxas de prevalência de LMERT, variando de $25 \%$ a $98 \%$. A localização mais frequentemente afetada é a coluna lombar sendo referenciada em 8 dos 21 artigos incluídos (Shieh, Sung, Su, Tsai, \& Hsieh, 2016; Machado, Rodrigues, Oliveira, Laudano, \& Sobrinho, 2014; Harcombe, Herbison, McBride, \& Derrett, 2014; Moreira, Sato, Foltran, Silva, \& Coury, 2014; Attar, 2014; Serralheira, Cotrim, Rodrigues, Nunes, \& Uva, 2012; Nasiri-Ziba, Nosrati, \& Hanani , 2016; Barzideh, Choobineh, \& Tabatabaee, 2014), seguindo-se a coluna cervical isoladamente mencionada em 4 artigos (Arvidsson, et al, 2016; Wang, Liu, Lu, \& Koo, 2015; Reed, Battistutta, Young, \& Newman, 2014; Long Johnston, \& Bogossian, 2013), coluna como um todo em 3 e, por fim, ombros e cervical em 2 dos artigos (Arvidsson et al., 2012; Simonsen, Arvidsson, \& Nordander, 2012). Os principais fatores de risco mencionados foram a idade, cansaço matinal, escassez de funcionários e/ou equipamentos, rácios elevados de utentes e queixas prévias de saúde dos enfermeiros. Foi referido que com o avançar da idade aumenta em $3 \%$ a probabilidade de ser desenvolvida uma LMERT e as queixas prévias aumentam esta mesma probabilidade para 5\% (Munabi et al., 2014). Os mesmos autores evidenciam uma maior percentagem de LMERT nos hospitais públicos comparativamente aos privados que relacionam com a escassez de funcionários e /ou equipamentos e com os rácios elevados nas instituições públicas. $\mathrm{O}$ artigo de Freimann et al. (2013) reforça que a idade é um fator de risco, além de fazer também referência à exaustão emocional. A relação entre a idade e a prevalência das LMERT aumenta em $75,5 \%$ quando a idade é igual ou superior a 35 anos (Shieh et al., 2016). Este artigo acrescenta como fator de risco a média de horas no trabalho e o tempo de permanência na posição de pé ou a andar. A obesidade, a fraca saúde física geral e o trabalho em unidades de terapia intensiva também foram considerados fatores de risco para o desenvolvimento das LMERT (Reed et al., 2014). No que se refere aos fatores de risco psicossociais foram referidos o stress, ritmo de trabalho acelerado, baixa satisfação e pouco respeito e justiça no trabalho, conduzindo também ao aparecimento de LMERT (Freimann et al., 2016). Constatou-se que a taxa de prevalência tem uma correlação direta com as atividades desenvolvidas, sendo mais elevada nas atividades de grande esforço físico, nomeadamente nos posicionamentos, mobilizações, transferências e higienização no leito, agravando-se quanto maior a dependência dos doentes (Alperovitch-Najenson et al., 2014; Serralheira et al., 2012). Trabalhar com posturas incorretas aumenta o risco de lesões cervicais em 35\% e lesões dorsais em 50\% (Long et al., 2013). As lesões lombares estão associadas a flexão do tronco, posturas estáticas e tarefas repetitivas, enquanto que as lesões cervicais e ombros estão associados ao aumento da carga física (Alperovitch-Najenson et al., 2014). Os autores Moreira et al. (2014) reforçam que as LMERT da coluna lombar estão associadas a posturas inadequadas no trabalho. As lesões exclusivas da coluna cervical devem-se à carga física no trabalho, posição inadequada prolongada com a 
cabeça fletida e o aumento da velocidade dos movimentos (Arvidsson et al., 2016). Reforçando que a maior parte das LMERT estão relacionadas com questões posturais, Arvidsson et al. (2012) mencionam no seu artigo que as enfermeiras do bloco operatório desenvolvem as LMERT por permanecerem um tempo prolongado em posições estáticas.

\section{DISCUSSÃO}

Os enfermeiros são um dos grupos profissionais a quem foi reconhecido, quer a nível nacional como internacional, elevadas taxas de LMERT e têm, por isso, sido alvo de mais investigação nesta área (Fernandes et al., 2018; Freimann et al., 2013).

Shieh et al., (2016) reforçam esta ideia afirmando que se trata de uma categoria profissional na área da saúde com maior risco para desenvolver este tipo de patologias. Constatou-se algum viés na seleção das amostras, nomeadamente amostras que excluíam o sexo masculino, designadamente nos estudos de Arvidsson et al. (2016), Arvidsson I. et al. (2012), Simonsen et al. (2012) e Alperovitch-Najenson et al. (2014), ou que impunham uma faixa etária (Wang et al., 2015; Freimann et al., 2013). Analisaram-se vários estudos comparativos da categoria profissional de enfermagem com outras categorias profissionais o que evidenciou que os enfermeiros apresentam maiores taxas de prevalência de LMERT. A comparação também permitiu a compreensão das diferenças das LMERT no que diz respeito à sua localização anatómica, que no caso de enfermagem tem como maior prevalência a lombalgia pela sua significativa associação com as atividades físicas como o levantamento de pesos $\geq 25 \mathrm{~kg}$ (Freimann et al., 2013). Outro aspeto importante a apontar é o facto de muitos enfermeiros não referirem ou relatarem as LMERT, chegando mesmo a não procurar ajuda (Wang et al., 2015). Muitas vezes automedicam-se como alternativa sendo que segundo o mesmo autor, 7,7\% dos enfermeiros o fizeram na semana antes do estudo. Tal realidade leva a que as taxas de prevalência reportadas possam estar subestimadas. A grande parte das LMERT centra-se nas lombalgias, porém a elevada prevalência também se consta em outras regiões anatómicas, podendo a percentagem não corresponder ao valor real nestas regiões pelo facto dos enfermeiros não as reportarem (Harcombe et al., 2014).

Os enfermeiros executam muita carga física na sua prática diária, pelo grande número de atividades elaboradas junto dos doentes, levando a lesões recorrentes (Shieh et al., 2016). Estão constantemente expostos a trabalhos exaustivos e a tensão músculoesquelética e representam uma percentagem considerável de recursos humanos em qualquer instituição de saúde, pelo que o desenvolvimento de LMERT pode ter um impacto negativo, levando a limitações durante a execução de tarefas, ausências nos locais de trabalho e até mesmo pedidos de mobilidade para outros serviços (Attar, 2014). Em casos mais graves, chegam mesmo a abandonar a profissão por queixas álgicas crónicas ao nível da coluna vertebral, tendo sido referido por Fouts et al. (2015) que 12 a 18\% dos enfermeiros o fizeram. Reforçando esta realidade, estudos em 5 países que incluíram um número elevado de enfermeiros

(43 000), chegaram à conclusão que muitos destes profissionais tencionam abandonar o seu local de trabalho, devido às exigências psicológicas e físicas a que estão submetidos, tendo sido enunciado uma percentagem considerável, mais concretamente, de 
17 a 39\% (Freimann et al., 2013). Tais situações acabam por prejudicar não só a saúde destes profissionais, mas também se refletem negativamente na entidade patronal e na sociedade em geral (Freimann et al., 2016).

Os enfermeiros por estarem continuamente expostos a um ou mais fatores de risco apresentam elevada prevalência de LMERT, tendo grande impacto na vida social, profissional, económica e pessoal, refletindo-se na sociedade e em todos os ambientes em que o enfermeiro se insere.

Os resultados desta revisão vão ao encontro dos resultados previamente encontrados por Davis \& Kotowsky (2015) e de Lelis e seus colaboradores (2012), revisões estas realizadas com o foco em períodos de tempo anteriores aos desta revisão. Daqui se pode inferir que, pese embora vários estudos tenham sido desenvolvidos para a implementação de estratégias para a prevenção das LMERT, a sua prevalência continua elevada (Hoof et al., 2018). De facto, não foi encontrada forte evidência para intervenções dirigidas à prevenção ou tratamento das LMERT em enfermeiros, assim como a política generalizada de não levantamento de pesos e foco na técnica correta de levantamento de pesos não está suportada em evidência científica (Hoof et al., 2018), pelo que serão necessários ensaios clínicos randomizados para avaliar a eficácia das intervenções destinadas a prevenir e tratar as LMERT.

\section{CONCLUSÃO}

Em relação à questão que norteou esta investigação, tornou-se evidente que as LMERT constituem uma realidade dos profissionais de enfermagem, com elevada prevalência, com maior destaque nas lesões ao nível da coluna lombar. Encontrou-se uma grande quantidade de estudos na temática em causa, mas devido à diversidade de métodos de colheita e análise de dados utilizadas suscitou alguma dificuldade na interpretação dos resultados obtidos, sendo esta uma das limitações deste estudo. Identificam-se, no entanto, outras limitações: a pesquisa realizada apenas para texto integral disponível, considerando que pode existir evidência disponível nas publicações que não têm texto integral acessível; o facto de os estudos estarem relacionados com diferentes valências de prestação de cuidados, realizados em diferentes países, logo em diferentes contextos e realidades, não permitindo comparabilidade; e o grande número de artigos encontrados relacionados com a temática em estudo, mas que não foi possível aceder ao texto completo. De salientar a escassez de estudos em Portugal. Mesmo assim, foi francamente percetível e esclarecedor que existe uma elevada prevalência de LMERT no que concerne esta categoria profissional ao nível mundial.

Além disso, e tendo em conta os fatores de risco que estão na causa destas lesões e que foram enunciados nos diferentes estudos apresentados, entende-se que será necessário tomar considerações importantes na prevenção das lesões músculo-esqueléticas na atividade de enfermagem. A investigação nesta área deverá ser dirigida para a interpretação dos fatores causais e implementação de medidas preventivas/corretivas e a capacitação das instituições para a importância de ações a nível preventivo, comportamental e instituição de medidas ergonómicas. 


\section{REFERÊNCIAS BIBLIOGRÁFICAS}

Abedinia, R., Choobinehb, A.R., \& Hasanzadehc, J. (2015). Patient manual handling risk assessment among hospital nurses. Work, 50(4), 669-75. https://doi: 10.3233/WOR141826

Alperovitch-Najenson, D., Sheffer, D., Treger, I., Finkels, T., \& Kalichman, L. (2014). Rehabilitation versus Nursing Home Nurses' Low Back and Neck-Shoulder Complaints. Rehabilitation Nursing, 40(5), 286-93. https://doi: 10.1002/rnj.172

Arvidsson, I., Simonsen, J. G., Dahlqvist, C., Axmon, A., Karlson, B., Björk, J., Nordander, C. (2016). Cross-sectional associations between occupational factors and musculoskeletal pain in women teachers, nurses and sonographers. BMC Musculoskeletal Disorders, 17, 35. https://doi: 10.1186/s12891-016-0883-4

Arvidsson, I., Simonsen, J. G., Balogh, I., Hansson, G., Dahlqvist, C., Granqvist, L. ... Nordander, C. (2012). Discrepancies in pain presentation caused by adverse psychosocial conditions as compared to pain due to high physical workload?. Work, 41, 2472-2475. https://doi: 10.3233/WOR-2012-0483-2472

Attar, S. M. (2014). Frequency and risk factors of musculoskeletal pain in nurses at a tertiary centre in Jeddah, Saudi Arabia: a cross sectional study. BMC Research Notes, 7, 61. https://doi: 10.1186/1756-0500-7-61

Barzideh, M., Choobineh, A. R., \& Tabatabaee, H. R. (2014). Job stress dimensions and their relationship to musculoskeletal disorders in Iranian nurses. Work, 47(4), 423-9. https://doi: 10.3233/WOR-121585

Briggs, J. (2017). Checklist for Prevalence Studies. The Joanna Briggs Institute. Retirado de: http://joannabriggs.org/research/criticalappraisal-tools.html

Coggon, D., Ntani, G., Palmer, KT., Felli, VE., Harari, R., Barrero, LH. ... Gray, A. (2013). Disabling musculoskeletal pain in working populations: is it the job, the person, or the culture? Pain, 154(6), 856-63. https://doi: 10.1016/j.pain.2013.02.008

Davis, K. G., \& Kotowsky, S. E. (2015). Prevalence of Musculoskeletal Disorders for Nurses in Hospitals, Long-Term Care Facilities, and
Home Health Care: A Comprehensive Review. The Journal of the Human Factors and Ergonomics Society, 57(5), 754-92. https://doi: 10.1177/0018720815581933

Fernandes, C. N., Couto, G., Carvalho, R., Fernandes, D. G., Brito, L., Carvalho, P., \& Ferreira, P. F. (2018). "Risk observation in the handling of dependent patients in health professionals of a hospital unit". Nursing Practice Today 5 (4), 385-394.

http://dx.doi.org/10.18502/npt.v5i4.117

Fouts B. L., Serres J. L., Dukes S. F., Maupin G. M., Wade M. E., \& Pohlman D. M. (2015). Investigation of Self-Reported Musculoskeletal Injuries on Post-Deployment Health Assessment Forms for Aeromedical Evacuation Personnel. Military Medicine, 180(12), 1256-61. https://doi: 10.7205/MILMED-D-14-00606

Freimann, T., Pääsuke, M., \& Merisalu, E. (2016). Work-Related Psychosocial Factors and Mental Health Problems Associated with Musculoskeletal Pain in Nurses: A Cross Sectional Study. Pain Research and Management, 2016, 1-7. https://doi: $10.1155 / 2016 / 9361016$

Freimann, T., Coggon, D., Merisalu, E., Animägi, L., \& Pääsuke, M. (2013). Risk factors for musculoskeletal pain amongst nurses in Estonia: a cross-sectional study. $B M C$ Musculoskeletal Disorders, 14, 334. https://doi: 10.1186/1471-2474-14-334

Galvão, T., \& Pereira, M. (2014). Revisões sistemáticas da literatura: passos para a sua elaboração. Epidemiologia e Serviços de Saúde, 23 (1), 183$184 . \quad$ https://doi: 10.5123/S167949742014000100018

Harcombe H., Herbison, G. P., McBride, D., \& Derrett, S. (2014). Musculoskeletal disorders among nurses compared with two other occupational groups. Occupational Medicine, 64(8), 601-7. https://doi: 10.1093/occmed/kqu117

Hoof, WV., O'Sullivan, K., O'Keeffe, M., Verschueren, S., O'Sullivan, P., \& Dankaerts, W. (2018). The efficacy of interventions for low back pain in nurses: A systematic review. International Journal of Nursing Studies, 77, 222-231. https://doi: 10.1016/j.ijnurstu.2017.10.015

Jerónimo, J., \& Cruz, A. (2014) - Estudo da prevalência e fatores de risco de lesões músculo- 
esqueléticas ligadas ao trabalho em enfermeiros. Revista de investigação em Enfermagem, 9, 35-46. retirado de: http://www.sinaisvitais.pt/images/stories/Ri e/Rie9_Serie2.pdf

Lelis, C., Battaus, M., Freitas, F., Rocha, F., Marziale, M., \& Robazzi, M. (2012). Distúrbios osteomusculares relacionados ao trabalho em profissionais de enfermagem: revisão integrativa da literatura. Acta Paulista de Enfermagem, 25 (3), 477-482. https://doi: 10.1590/S0103-21002012000300025

Long, M., Bogossian, F., \& Johnston, V. (2013). The prevalence of work-related neck, shoulder and upper back musculoskeletal disorders among midwives, nurses, and physicians. A systematic review. Workplace Health \& Safety, 61(5), 223-229. https://doi: $10.1177 / 216507991306100506$

Long, M. H., Johnston, V., \& Bogossian, F. E. (2013). Helping women but hurting ourselves? Neck and upper back musculoskeletal symptoms in a cohort of Australian Midwives. Midwifery, 29(4), 359-67. https://doi: 10.1016/j.midw.2012.02.003

Machado, L. S., Rodrigues, E. P., Oliveira, L. M., Laudano R. C., \& Sobrinho, C. L. (2014). Health problems reported by nursing workers in a public hospital of Bahia. Revista Brasileira de Enfermagem, 67 (5), 684-91.

Moher, D., Shamseer, L., Clarke, M., Ghersi, D., Liberati, A., Petticrew, M., ... Group, P.-P. (2015). Preferred Reporting Items for Systematic Review and Meta-Analysis Protocols (PRISMA-P) 2015 statement. Systematic Reviews, 4, 9. https://doi: 10.1186/2046-4053-4-1

Moreira, R. F., Sato, T. O., Foltran, F. A., Silva, L. C., \& Coury, H. J. (2014). Prevalence of musculoskeletal symptoms in hospital nurse technicians and licensed practical nurses: associations with demographic factors. Brazilian Journal of Physical Therapy, 18(4), 323-333. https://doi: 10.1590/bjptrbf.2014.0026

Munabi, I. G., Buwembo, W., Kitara, D. L., Ochieng, J., Nabirye, R. C., \& Mwaka, E. S. (2014). Musculoskeletal disorders among nursing staff: a comparison of five hospitals in Uganda. The Pan African Medical Journal, 17, 81-8. https://doi: 10.11604/pamj.2014.17.81.3213
Nasiri-Ziba, F., Nosrati, S., Hanani, S. (2016). The prevalence of musculoskeletal disorders among undergraduates and technicians of operating room of the educational hospitals affiliated with Iran University of Medical Sciences in 2016. Nursing Practice Today, 4(3), 134-142. Retirado de: http://npt.tums.ac.ir/index.php/npt/article/v iew/251

Ranney, D. (2000). Distúrbios osteomusculares Crónicos Relacionados ao Trabalho. São Paulo: Editora Roca.

Reed, L. F., Battistutta, D., Young, J., \& Newman, B. (2014). Prevalence and risk factors for foot and ankle musculoskeletal disorders experienced by nurses. BMC Musculoskeletal Disorders, 15, 196. https://doi: 10.1186/14712474-15-196

Serralheira, F., \& Uva, A. (2007). Identificação e avaliação do risco de LMEMSLT. Livro de Atas da Conferência Internacional SHO 2007. Sociedade Portuguesa de Segurança e Higiene Ocupacionais: Guimarães. ISBN: 978-97299504-3-8

Serralheira, F., Cotrim, T., Rodrigues, V., Nunes, C., \& Uva, A. (2012). Lesões músculo-esqueléticas Ligadas ao trabalho em enfermeiros portugueses: "ossos do ofício» ou doenças relacionadas com o trabalho. Revista Portuguesa de Saúde Pública, 30 (2), 193-203. retirado de: http://www.scielo.mec.pt/pdf/rpsp/v30n2/v 30n2a10.pdf

Shieh, S. H., Sung, F. C., Su,C. H., Tsai, Y., \& Hsieh, V. C. (2016). Increased low back pain risk in nurses with high workload for patient care: A questionnaire survey. Taiwanese Journal of Obstetrics and Gynecology, 55(4), 525-9. https://doi: 10.1016/j.tjog

Simonsen, J. G., Arvidsson, I. \& ,Nordander, C. (2012). Ergonomics in the operating room. Work, 41 (Suppl 1), 5644-6. https://doi: 10.3233/WOR2012-0905-5644

Souza, M., Silva, M., \& Carvalho, R. (2010). Revisão integrativa: o que é e como fazer. Einstein, 8 102-108. http://dx.doi.org/10.1590/s167945082010rw1134 
Wang, S. Y., Liu, L. C., Lu, M. C., \& Koo, M. (2015). Comparisons of musculoskeletal disorders among ten different medical professions in Taiwan: a nationwide, population-based study. PLoS One, 10(4), e0123750. https://doi:

10.1371/journal.pone.0123750 